\title{
An elastic analysis for thick cylindrical pressure vessels with variable thickness
}

\author{
Mehdi Ghannad ${ }^{\mathrm{a}}$, Mehdi Jabbari ${ }^{\mathrm{b}}$ and Mohammad Zamani Nejad ${ }^{\mathrm{b} *}$
}

${ }^{a}$ Mechanical Mechanical Engineering Faculty, Shahrood University, Shahrood, Iran ${ }^{b}$ Mechanical Engineering Department, Yasouj University, Yasouj, Iran

\begin{tabular}{|c|c|}
\hline $\begin{array}{ll}\text { A RT I C L E INFO } & \text { I F }\end{array}$ & A B S T RA C T \\
\hline $\begin{array}{l}\text { Article history: } \\
\text { Received October 6, } 2014 \\
\text { Accepted } 28 \text { January } 2015 \\
\text { Available online } \\
29 \text { January } 2015 \\
\text { Keywords: } \\
\text { Thick cylindrical shell with } \\
\text { variable thickness } \\
\text { Disk form multilayers } \\
\text { Matched asymptotic method } \\
\text { Finite element method }\end{array}$ & $\begin{array}{l}\text { This paper derives a semi-analytical solution to determine displacements and stresses in a thick } \\
\text { cylindrical shell with variable thickness under uniform pressure based on disk form } \\
\text { multilayers. The proposed study partitions the thick cylinder into disk-layer parts based on their } \\
\text { thickness of the cylinder. According to the existence of shear stress in the thick cylindrical } \\
\text { shell with variable thickness, the equations governing disk layers are acquired based on first } \\
\text { shear deformation theory (FSDT), which are in the form of a set of general differential } \\
\text { equations. In this study, the cylinder is partitioned into } n \text { different disks and } n \text { sets of differential } \\
\text { equations are derived. The solution of these equations provides displacements and stresses } \\
\text { based on the boundary conditions and continuity conditions between the layers. The results are } \\
\text { compared with those obtained through the analytical solution and the numerical solution. For } \\
\text { the purpose of the analytical solution, matched asymptotic method (MAM) and for the } \\
\text { analytical solution, the finite element method (FEM are implemented. }\end{array}$ \\
\hline
\end{tabular}

\section{Introduction}

Thick cylindrical shells with variable thickness have extensively been used in various fields such as space flight, aviation, rocket and submarine technology. Given the limitations of the classic theories of thick wall shells, there has been few works associated with the analytical and semi-analytical solutions of these shells (Talaee et al., 2014). Naghdi and Cooper (1956) formulated the theory of shear deformation by considering the transverse shear effect. Mirsky and Hermann (1956) investigated the solution of thick cylindrical shells of homogenous and isotropic materials, using the first-order shear deformation theory (FSDT). Greenspon (1960) compared between the findings regarding various solutions obtained for cylindrical shells. Vekua (1965) built a refined theory for shallow shells with variable thickness. Suzuki et al. (1981) studied the axisymmetric vibrations of a cylindrical shell where the thickness differs in the axial direction by using the thin cylindrical shell theory and an improved

* Corresponding author. Tel/Fax: +98 7433221711

E-mail addresses: m.zamani.n@gmail.com, m_zamani@yu.ac.ir (M. Zamani Nejad) 
thick cylindrical shell theory by applying a series solution. Kang and Leissa (2001) performed an investigation where equations of motion and energy functional were derived for a three-dimensional coordinate system. The field equations were utilized to express them in terms of displacement components. Eipakchi et al. (2003) implemented the FSDT in order to find governing equations of thick cylinders with varying thickness and analyzed the equations based on perturbation theory. Using tensor analysis, a complete 3-D set of field equations was proposed for elastic analysis of thick shells of revolution with arbitrary curvature and variable thickness along the meridional direction made of functionally graded materials by Nejad et al. (2009). Ghannad et al. (2009) proposed to use the FSDT analytical solution for homogeneous and isotropic truncated thick conical shell. Ghannad and Nejad (2010) calculated the differential equations governing the homogenous and isotropic axisymmetric thick-walled cylinders with same boundary conditions at the two ends, based on the first-order shear deformation theory and the virtual work principle. In addition, they also solved the set of nonhomogenous linear differential equations for the cylinder with clamped-clamped ends. Finally, Ghannad et al. (2012) presented an analytical solution for clamped-clamped thick cylindrical shells with variable thickness by considering constant internal pressure. In this paper, elastic analysis is presented for pressurized thick cylindrical shells with variable thickness using disk form multilayers.

\section{Formulation of problem}

According to the first-order shear deformation theory, the parts, which are straight and perpendicular to the mid-plane stay straight but not always perpendicular after deformation and loading. In such circumstances, shear strain and shear stress are considered. Fig. 1 shows geometry of a thick cylindrical shell where $h$ and $L$ represent variable thickness and length, respectively.

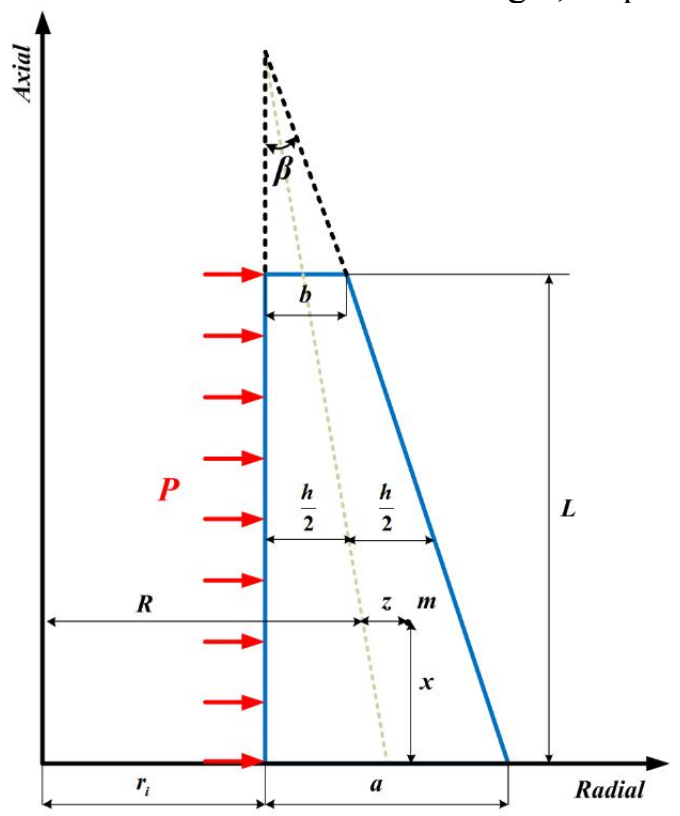

Fig. 1. Thick cylindrical shell with variable thickness

The location of a typical point $m$, within the shell element is as follows,

$$
\left\{\begin{array}{l}
m:(r, x)=(R+z, x) \\
0 \leq x \leq L \quad \& \quad-\frac{h}{2} \leq z \leq \frac{h}{2}
\end{array}\right.
$$

where $z$ is the distance of typical point from the middle surface. In Eq. (1), $R$ and variable thickness $h$ are 


$$
\left\{\begin{array}{l}
R(x)=r_{i}+\frac{a}{2}-\frac{1}{2}(\tan \beta) x \\
h(x)=r_{i}+a-(\tan \beta) x
\end{array}\right.
$$

where $\beta$ is tapering angle as

$$
\beta=\tan ^{-1}\left(\frac{a-b}{L}\right)
$$

The general axisymmetric displacement field $\left(U_{x}, U_{z}\right)$, in the first-order Mirsky-Hermann's theory (1956) could be stated on the basis of axial displacement and radial displacement, as follows,

$$
\left\{\begin{array}{l}
U_{x}(x, z)=u(x)+\phi(x) z \\
U_{z}(x, z)=w(x)+\psi(x) z
\end{array}\right.
$$

where $u(x)$ and $w(x)$ are the displacement components of the middle surface. Also, $\phi(x)$ and $\psi(x)$ are the functions applied to determine the displacement field. The kinematic equations (straindisplacement relations) in the cylindrical coordinates system are

$$
\left\{\begin{array}{l}
\varepsilon_{x}=\frac{\partial U_{x}}{\partial x}=\frac{d u}{d x}+\frac{d \phi}{d x} z \\
\varepsilon_{\theta}=\frac{U_{z}}{r}=\left(\frac{w}{R+z}\right)+\left(\frac{\psi}{R+z}\right) z \\
\varepsilon_{z}=\frac{\partial U_{z}}{\partial z}=\psi \\
\gamma_{x z}=\frac{\partial U_{x}}{\partial z}+\frac{\partial U_{z}}{\partial x}=\left(\phi+\frac{d w}{d x}\right)+\frac{d \psi}{d x} z
\end{array}\right.
$$

The stress-strain relations (constitutive equations) for homogeneous and isotropic materials are as follows,

$$
\left\{\begin{array}{l}
\sigma_{x} \\
\sigma_{\theta} \\
\sigma_{z} \\
\tau_{x z}
\end{array}\right\}=\lambda\left[\begin{array}{cccc}
1-v & v & v & 0 \\
v & 1-v & v & 0 \\
v & v & 1-v & 0 \\
0 & 0 & 0 & \frac{1-2 v}{2}
\end{array}\right]\left\{\begin{array}{c}
\varepsilon_{x} \\
\varepsilon_{\theta} \\
\varepsilon_{z} \\
\gamma_{x z}
\end{array}\right\}
$$

where $\sigma_{i}$ and $\varepsilon_{i}, i=x, \theta, z$ are the stresses and strains in the axial $(x)$, circumferential $(\theta)$, and radial $(z)$ directions. $v$ and $E$ are Poisson's ratio and modulus of elasticity, respectively. In Eqs. (6), $\lambda$ is

$$
\lambda=\frac{E}{(1+v)(1-2 v)} .
$$

The normal forces $\left(N_{x}, N_{\theta}, N_{z}\right)$, bending moments $\left(M_{x}, M_{\theta}, M_{z}\right)$, shear force $\left(Q_{x}\right)$, and the torsional moment $\left(M_{x z}\right)$ in terms of stress resultants are 


$$
\begin{aligned}
& \left\{\begin{array}{l}
N_{x} \\
N_{\theta} \\
N_{z}
\end{array}\right\}=\int_{-h / 2}^{h / 2}\left\{\begin{array}{c}
\sigma_{x}\left(1+\frac{z}{R}\right) \\
\sigma_{\theta} \\
\sigma_{z}\left(1+\frac{z}{R}\right)
\end{array}\right\} d z \\
& \left\{\begin{array}{l}
M_{x} \\
M_{\theta} \\
M_{z}
\end{array}\right\}=\int_{-h / 2}^{h / 2}\left\{\begin{array}{c}
\sigma_{x}\left(1+\frac{z}{R}\right) \\
\sigma_{\theta} \\
\sigma_{z}\left(1+\frac{z}{R}\right)
\end{array}\right\} z d z \\
& Q_{x}=K \int_{-h / 2}^{h / 2} \tau_{x z}\left(1+\frac{z}{R}\right) d z \\
& M_{x z}=K \int_{-h / 2}^{h / 2} \tau_{x z}\left(1+\frac{z}{R}\right) z d z
\end{aligned}
$$

where $K$ is the shear correction factor that is embedded in the shear stress term. In the static state, $K=5 / 6$ for cylindrical shells (Vlachoutsis, 1992). On the basis of the principle of virtual work, the variations of strain energy are equal to the variations of work of external forces as follows;

$$
\delta U=\delta W
$$

where $U$ is the total strain energy of the elastic body and $W$ is the total work of external forces due to internal pressure $P$. With substituting strain energy and work of external forces, we have,

$$
\int_{0}^{L} R(x) \int_{-h / 2}^{h / 2}\left(\sigma_{x} \delta \varepsilon_{x}+\sigma_{\theta} \delta \varepsilon_{\theta}+\sigma_{z} \delta \varepsilon_{z}+\tau_{x z} \delta \gamma_{x z}\right)\left(1+\frac{z}{R}\right) d z d x=\int_{0}^{L} P \delta U_{z}\left(R-\frac{h}{2}\right) d x .
$$

Substituting Eqs. (5) and (6) into Eq. (13), and drawing upon calculus of variation and the virtual work principle, we will have,

$$
\left\{\begin{array}{l}
N_{x} R=C_{0} \\
M_{x} \frac{d R}{d x}+R\left(\frac{d M_{x}}{d x}-Q_{x}\right)=0 \\
Q_{x} \frac{d R}{d x}+R\left(\frac{d Q_{x}}{d x}\right)-N_{\theta}=-P\left(R-\frac{h}{2}\right) \\
M_{x z} \frac{d R}{d x}+R\left(\frac{d M_{x z}}{d x}-N_{z}\right)-M_{\theta}=P \frac{h}{2}\left(R-\frac{h}{2}\right)
\end{array}\right.
$$

and the boundary conditions are

$$
\left[\left(N_{x} \delta u+M_{x} \delta \phi+Q_{x} \delta w+M_{x z} \delta \psi\right) R\right]_{0}^{L}=0
$$

Eq. (15) states the boundary conditions which must exist at the two ends of the cylinder. In order to solve the set of differential equations (14), with using of Eqs. (5) to (11), and then using Eq. (14), we have 


$$
\begin{aligned}
& \left\{\left[B_{1}\right] \frac{d^{2}}{d x^{2}}\{y\}+\left[B_{2}\right] \frac{d}{d x}\{y\}+\left[B_{3}\right]\{y\}=\{F\}\right. \\
& \{y\}=\{d u / d x \quad \phi \quad w \quad \psi\}^{T}
\end{aligned}
$$

The coefficients matrices $\left[B_{i}\right]_{4 \times 4}$, and force vector $\{F\}_{4 \times 1}$ are as follows,

$$
\begin{aligned}
& {\left[B_{1}\right]=\left[\begin{array}{cccc}
0 & 0 & 0 & 0 \\
0 & (1-v) \frac{h^{3}}{12} R & 0 & 0 \\
0 & 0 & \mu h R & \frac{\mu h^{3}}{12} \\
0 & 0 & \frac{\mu h^{3}}{12} & \frac{\mu h^{3}}{12} R
\end{array}\right]} \\
& {\left[B_{2}\right]=\left[\begin{array}{cccc}
0 & (1-v) \frac{h^{3}}{12} & 0 & 0 \\
(1-v) \frac{h^{3}}{12} & (1-v) \frac{h^{2}}{12}\left(3 R \frac{d h}{d x}+h \frac{d R}{d x}\right) & -\mu h R & -(\mu-2 v) \frac{h^{3}}{12} \\
0 & \mu h R & \mu\left(R \frac{d h}{d x}+h \frac{d R}{d x}\right) & \frac{\mu h^{2}}{4} \frac{d h}{d x} \\
0 & (\mu-2 v) \frac{h^{3}}{12} & \frac{\mu h^{2}}{4} \frac{d h}{d x} & \frac{\mu h^{2}}{12}\left(3 R \frac{d h}{d x}+h \frac{d R}{d x}\right)
\end{array}\right]} \\
& {\left[B_{3}\right]=\left[\begin{array}{cccc}
(1-v) h R & 0 & v h & v h R \\
(1-v) \frac{h^{2}}{4} \frac{d h}{d x} & -\mu h R & 0 & \frac{v h^{2}}{2} \frac{d h}{d x} \\
-v h & \mu\left(R \frac{d h}{d x}+h \frac{d R}{d x}\right) & -(1-v) \alpha & -h+(1-v) \alpha R \\
-v h R & \frac{\mu h^{2}}{4} \frac{d h}{d x} & -h+(1-v) \alpha R & -(1-v) \alpha R^{2}
\end{array}\right]} \\
& \{F\}=\frac{1}{\lambda}\left\{\begin{array}{c}
C_{0} \\
0 \\
-P\left(R-\frac{h}{2}\right) \\
P \frac{h}{2}\left(R-\frac{h}{2}\right)
\end{array}\right\}
\end{aligned}
$$

where the parameters are as follows,

$$
\left\{\begin{array}{l}
\mu=\frac{5}{12}(1-2 v) \\
\alpha=\ln \left(\frac{R+h / 2}{R-h / 2}\right)
\end{array}\right.
$$


The set of differential Eqs. (16) has been solved by perturbation technique (Ghannad \& Nejad 2010). In the next section, a new method is presented for solving set of Eqs. (16).

\section{Solution with disk form multilayers}

In this technique, the thick cylinder with variable thickness is divided to disk layers with constant height $h$ as shown in Fig. 2. Therefore, the governing equations are converted to nonhomogeneous set of differential equations with constant coefficients. $x^{[k]}$ and $R^{[k]}$ are length and radius of middle of disks, respectively and $k$ is the number of disks. The modulus of elasticity and Poisson's ratio of disks are assumed to be constant. The length of middle of an arbitrary disk shown Fig. 3 is as follows,

$$
\left\{\begin{array}{l}
x^{[k]}=\left(k-\frac{1}{2}\right) \frac{L}{n} \\
\left(x^{[k]}-\frac{t}{2}\right) \leq x \leq\left(x^{[k]}+\frac{t}{2}\right) \\
t=\frac{L}{n}
\end{array}\right.
$$

where $n$ is the number of disks and $k$ is the corresponding number given to each disk.
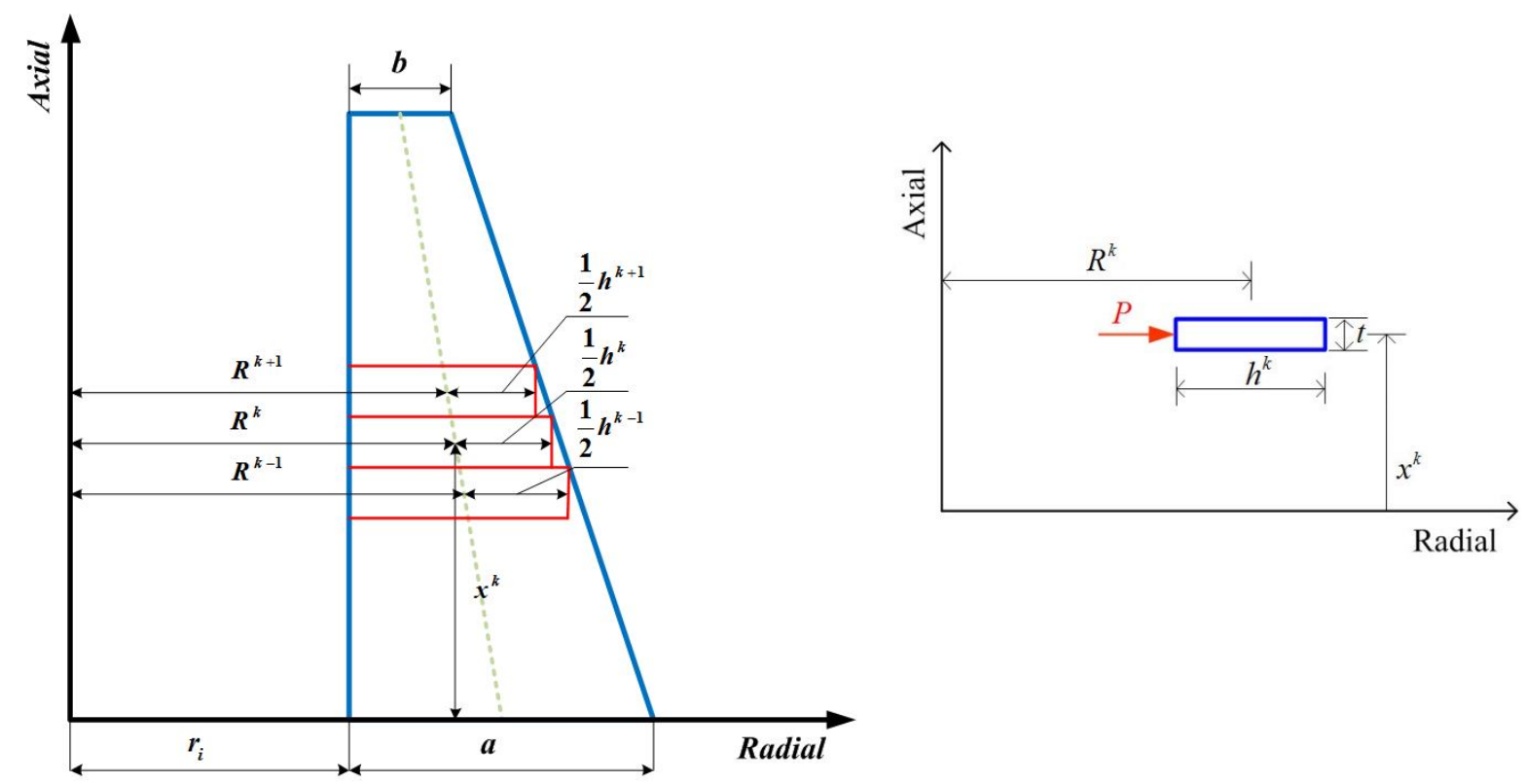

Fig. 2. Dividing of thick cylinder with variable Fig. 3. Geometry of an arbitrary disk layer thickness to disk form multilayer

The radius of middle point of each disk is as follows,

$$
\left\{\begin{array}{l}
R^{[k]}=r_{i}+\frac{h^{[k]}}{2} \\
h^{[k]}=a-\tan (\beta) x^{[k]}
\end{array}\right.
$$

Thus

$$
\left(\frac{d h}{d x}\right)^{[k]}=2\left(\frac{d R}{d x}\right)^{[k]}=-\tan \beta
$$


By considering shear stress and based on FSDT, nonhomogeneous set of ordinary differential equations with constant coefficient of each disk is obtained.

$$
\left\{\begin{array}{l}
{\left[B_{1}\right]^{[k]} \frac{d^{2}}{d x^{2}}\{y\}^{[k]}+\left[B_{2}\right]^{[k]} \frac{d}{d x}\{y\}^{[k]}+\left[B_{3}\right]^{[k]}\{y\}^{[k]}=\{F\}^{[k]}} \\
\{y\}^{[k]}=\left\{(d u / d x)^{[k]} \quad \phi^{[k]} \quad w^{[k]} \psi^{[k]}\right\}^{T}
\end{array}\right.
$$

The coefficients matrices $\left[B_{i}\right]_{4 \times 4}^{[k]}$, and force vector $\{F\}_{4 \times 1}^{k}$ are as follows,

$$
\begin{aligned}
& {\left[B_{1}\right]^{[k]}=\left[\begin{array}{cccc}
0 & 0 & 0 & 0 \\
0 & (1-v) \frac{\left(h^{[k]}\right)^{3}}{12} R^{[k]} & 0 & 0 \\
0 & 0 & \mu h^{[k]} R^{[k]} & \frac{\mu\left(h^{[k]}\right)^{3}}{12} \\
0 & 0 & \frac{\mu\left(h^{[k]}\right)^{3}}{12} & \frac{\mu\left(h^{[k]}\right)^{3}}{12} R^{[k]}
\end{array}\right]} \\
& {\left[\begin{array}{ll}
0 & (1-v) \frac{\left(h^{[k]}\right)^{3}}{12}
\end{array}\right.} \\
& {\left[B_{2}\right]^{[k]}=\left[\begin{array}{cc}
(1-v) \frac{\left(h^{[k]}\right)^{3}}{12} & -(1-v) \frac{\left(h^{[k]}\right)^{2}}{24}\left(6 R^{[k]}+h^{k k}\right. \\
0 & \mu h^{[k]} R^{[k]} \\
0 & (\mu-2 v) \frac{\left(h^{[k]}\right)^{3}}{12}
\end{array}\right.}
\end{aligned}
$$

$$
\left.\begin{array}{cc}
0 & 0 \\
-\mu h^{[k]} R^{[k]} & -(\mu-2 v) \frac{\left(h^{[k]}\right)^{3}}{12} \\
-\mu\left(R^{[k]}+\frac{h^{[k]}}{2}\right) \tan \beta & -\frac{\mu\left(h^{[k]}\right)^{2}}{4} \tan \beta \\
-\frac{\mu\left(h^{[k]}\right)^{2}}{4} \tan \beta & -\frac{\mu\left(h^{[k]}\right)^{2}}{24}\left(6 R^{[k]}+h^{[k]}\right) \tan \beta
\end{array}\right]
$$




$$
\begin{aligned}
& {\left[B_{3}\right]^{[k]}=\left[\begin{array}{cc}
(1-v) h^{[k]} R^{[k]} & 0 \\
-(1-v) \frac{\left(h^{[k]}\right)^{2}}{4} \tan \beta & -\mu h^{[k]} R^{[k]} \\
-v h^{[k]} & -\mu\left(R^{[k]}+\frac{h^{[k]}}{2}\right) \tan \beta \\
-v h^{[k]} R^{[k]} & -\frac{\mu\left(h^{[k]}\right)^{2}}{4} \tan \beta
\end{array}\right.} \\
& \begin{array}{cc}
v h^{[k]} & v h^{[k]} R^{[k]} \\
0 & -\frac{v\left(h^{[k]}\right)^{2}}{2} \tan \beta
\end{array} \\
& -(1-v) \alpha^{[k]} \quad-h^{[k]}+(1-v) \alpha^{[k]} R^{[k]} \\
& -h^{[k]}+(1-v) \alpha^{[k]} R^{[k]} \quad-(1-v) \alpha^{[k]}\left(R^{[k]}\right)^{2} \\
& \{F\}^{[k]}=\frac{P\left(R^{[k]}-\frac{h^{[k]}}{2}\right)}{\lambda}\left\{\begin{array}{c}
C_{0} \\
0 \\
-1 \\
\frac{h^{[k]}}{2}
\end{array}\right\}
\end{aligned}
$$

where the parameters are as follows,

$$
\left\{\begin{array}{l}
\mu=\frac{5}{12}(1-2 v) \\
\alpha^{[k]}=\ln \left(\frac{R^{[k]}+h^{[k]} / 2}{R^{[k]}-h^{[k]} / 2}\right)
\end{array}\right.
$$

Defining the differential operator $P(D)$, Eq. (25) is written as follows,

$$
\left\{\begin{array}{l}
{[P(D)]^{[k]}=\left[B_{1}\right]^{[k]} D^{2}+\left[B_{2}\right]^{[k]} D+\left[B_{3}\right]^{[k]}} \\
D^{2}=\frac{d^{2}}{d x^{2}} \quad, \quad D=\frac{d}{d x}
\end{array}\right.
$$

Thus,

$$
[P(D)]^{[k]}\{y\}^{[k]}=\{F\}^{[k]}
$$

The above differential Equation has the total solution including general solution for homogeneous case $\{y\}_{h}^{[k]}$ and particular solution $\{y\}_{p}^{[k]}$, as follows:

$$
\{y\}^{[k]}=\{y\}_{h}^{[k]}+\{y\}_{p}^{[k]}
$$


For the general solution for homogeneous case, $\{y\}_{h}^{[k]}=\{V\}^{[k]} e^{m^{[k]} x}$ is substituted in

$$
\begin{aligned}
& {[P(D)]^{[k]}\{y\}^{[k]}=0 .} \\
& \left|m^{2}\left[B_{1}\right]^{[k]}+m\left[B_{2}\right]^{[k]}+\left[B_{3}\right]^{[k]}\right|=0
\end{aligned}
$$

Thus

$$
\begin{aligned}
& \mid \begin{array}{llll}
B_{11} & B_{12} & B_{13} & B_{14} \\
B &
\end{array} \\
& B_{21} \quad B_{22} \quad B_{23} \quad B_{24}=0 \\
& B_{31} \quad B_{32} \quad B_{33} \quad B_{34}=0 \\
& \left|\begin{array}{llll}
B_{41} & B_{42} & B_{43} & B_{44}
\end{array}\right| \\
& B_{11}=(1-v) h^{[k]} R^{[k]} \\
& B_{12}=m(1-v) \frac{\left(h^{[k]}\right)^{3}}{12} \\
& B_{13}=-B_{31}=v h^{[k]} \\
& B_{14}=-B_{41}=v h^{[k]} R^{[k]} \\
& B_{21}=(1-v) \frac{\left(h^{[k]}\right)^{2}}{12}\left(m h^{[k]}-3 \tan \beta\right) \\
& B_{22}=(1-v) \frac{\left(h^{[k]}\right)^{2}}{24}\left[2 m^{2} R^{[k]} h^{[k]}-m\left(6 R^{[k]}+h^{[k]}\right) \tan \beta\right]-\mu h^{[k]} R^{[k]} \\
& B_{23}=-m \mu h^{[k]} R^{[k]} \\
& B_{24}=-\frac{\left(h^{[k]}\right)^{2}}{12}\left[m(\mu-2 v) h^{[k]}+6 v \tan \beta\right] \\
& B_{32}=\mu\left[m h^{[k]} R^{[k]}-\left(R^{[k]}+\frac{h^{[k]}}{2}\right) \tan \beta\right] \\
& B_{33}=\mu\left[m^{2} h^{[k]} R^{[k]}-m\left(R^{[k]}+\frac{h^{[k]}}{2}\right) \tan \beta\right]-(1-v) \alpha^{[k]} \\
& B_{34}=B_{43}=\frac{\mu\left(h^{[k]}\right)^{2}}{12}\left[m^{2}\left(h^{[k]}\right)-3 m \tan \beta\right]-\left(h^{[k]}-(1-v) \alpha^{[k]} R^{[k]}\right) \\
& B_{42}=\frac{\left(h^{[k]}\right)^{2}}{12}\left(m(\mu-2 v) h^{[k]}-3 \mu \tan \beta\right) \\
& B_{44}=\frac{\mu\left(h^{[k]}\right)^{2}}{24}\left[2 m^{2} h^{[k]} R^{[k]}-m\left(6 R^{[k]}+h^{[k]}\right) \tan \beta\right]-(1-v) \alpha^{[k]}\left(R^{[k]}\right)^{2}
\end{aligned}
$$

The result of the determinant above is a six-order polynomial which is a function of $m$, the solution of which is a 6 eigenvalues $m_{i}$. The eigenvalues are 3 pairs of conjugated root. Substituting the calculated eigenvalues in following equation, the corresponding eigenvectors $\{V\}_{i}$ are obtained. 
$\left[m^{2}\left[B_{1}\right]^{[k]}+m\left[B_{2}\right]^{[k]}+\left[B_{3}\right]^{[k]}\right]\{V\}^{[k]}=0$.

Therefore, the homogeneous solution for is

$\{y\}_{h}^{[k]}=\sum_{i=1}^{6} C_{i}^{[k]}\{V\}_{i}^{[k]} e^{m_{i}^{[k]} x}$

The particular solution is obtained as follows.

$\{y\}_{p}^{[k]}=\left[\left[B_{3}\right]^{[k]}\right]^{-1}\{F\}^{[k]}$

Therefore, the total solution for is follows,

$\{y\}^{[k]}=\sum_{i=1}^{6} C_{i}\{V\}_{i}^{[k]} e^{m_{i}^{[k]} x}+\left[\left[B_{3}\right]^{[k]}\right]^{-1}\{F\}^{[k]}$

In general, the problem for each disk consists of 8 unknown values of $C_{i}$, including $C_{0}$ (first relation of Eq. 14), $C_{1}$ to $C_{6}$ (Eq. 52), and $C_{7}$ (Eq. $u^{[k]}=\int(d u / d x)^{[k]} d x+C_{7}$ ).

\section{Boundary and continuity conditions}

In this problem, the boundary conditions of cylinder is clamped-clamped ends, then we have,

$$
\left\{\begin{array}{c}
u \\
\phi \\
w \\
\psi
\end{array}\right\}_{x=0}=\left\{\begin{array}{c}
u \\
\phi \\
w \\
\psi
\end{array}\right\}_{x=L}=\left\{\begin{array}{l}
0 \\
0 \\
0 \\
0
\end{array}\right\}
$$

Therefore,

$$
\left\{\begin{array}{l}
U_{x}(x, z) \\
U_{z}(x, z)
\end{array}\right\}_{x=0, L}=\left\{\begin{array}{l}
0 \\
0
\end{array}\right\}
$$

According to continuity and homogeneity of the cylinder, at the boundary between two layers, forces, stresses and displacements must be continuous. Given that the applied shear deformation theory is an approximation of one order and also all equations related to the stresses include the first derivatives of displacement, the continuity conditions are as follows,

$$
\begin{aligned}
& \left\{\begin{array}{l}
U_{x}^{[k-1]}(x, z) \\
U_{z}^{[k-1]}(x, z)
\end{array}\right\}_{x=x^{[k-1]}+\frac{t}{2}}=\left\{\begin{array}{l}
U_{x}^{[k]}(x, z) \\
U_{z}^{[k]}(x, z)
\end{array}\right\}_{x=x^{[k]}-\frac{t}{2}} \\
& \left\{\begin{array}{l}
U_{x}^{[k]}(x, z) \\
U_{z}^{[k]}(x, z)
\end{array}\right\}_{x=x^{[k]}+\frac{t}{2}}=\left\{\begin{array}{l}
U_{x}^{[k+1]}(x, z) \\
U_{z}^{[k+1]}(x, z)
\end{array}\right\}_{x=x^{[k+1]}-\frac{t}{2}}
\end{aligned}
$$

and

$$
\left\{\begin{array}{l}
\frac{d U_{x}^{[k-1]}(x, z)}{d x} \\
\frac{d U_{z}^{[k-1]}(x, z)}{d x}
\end{array}\right\}_{x=x^{[k-1]}+\frac{t}{2}}=\left\{\begin{array}{l}
\frac{d U_{x}^{[k]}(x, z)}{d x} \\
\frac{d U_{z}^{[k]}(x, z)}{d x}
\end{array}\right\}_{x=x^{[k]}-\frac{t}{2}}
$$




$$
\left\{\begin{array}{l}
\frac{d U_{x}^{[k]}(x, z)}{d x} \\
\frac{d U_{z}^{[k]}(x, z)}{d x}
\end{array}\right\}_{x=x^{[k]}+\frac{t}{2}}=\left\{\begin{array}{l}
\frac{d U_{x}^{[k+1]}(x, z)}{d x} \\
\frac{d U_{z}^{[k+1]}(x, z)}{d x}
\end{array}\right\}_{x=x^{[k+1]}-\frac{t}{2}}
$$

Given the continuity conditions, in terms of $z, 8$ equations are achieved. In general, if the cylinder is divided into $n$ disk layers, $8(n-1)$ equations are obtained. Using the 8 equations of boundary condition, $8 n$ equations are obtained. The solution of these equations yields $8 n$ unknown constants.

\section{Results and Discussion}

A cylindrical shell with $r_{i}=40 \mathrm{~mm}, a=20 \mathrm{~mm}, b=10 \mathrm{~mm}$, and $L=800 \mathrm{~mm}$ will be considered in this paper. For analytical and numerical results the properties used are $E=200 \mathrm{GPa}$ and $v=0.3$. The applied internal pressure is $80 \mathrm{MPa}$. The thick cylindrical shell with variable thickness has clamped-clamped boundary conditions.

The effect of the number of disk layers on the radial displacement is presented in Fig. 4. It is observed that if number of disk layers is fewer than 50, it will have a substantial impact on the response. However, if the number of layers is above 60 disks, there will be no substantial impact on radial displacement. In the problem in question 75 disks are applied. Fig. 5 shows the distribution of axial displacement at different layers. At points away from the boundaries, axial displacement does not show significant differences in different layers, while at points near the boundaries, the reverse holds true. The distribution of radial displacement at various layers is plotted in Fig. 6 . The radial displacement at points away from the boundaries depends on radius and length. According to Figs. 5 and 6, the change in axial and radial displacements in the lower boundary is greater than that of the upper boundary and the greatest axial and radial displacement occurs in the internal surface $(z=-h / 2)$.
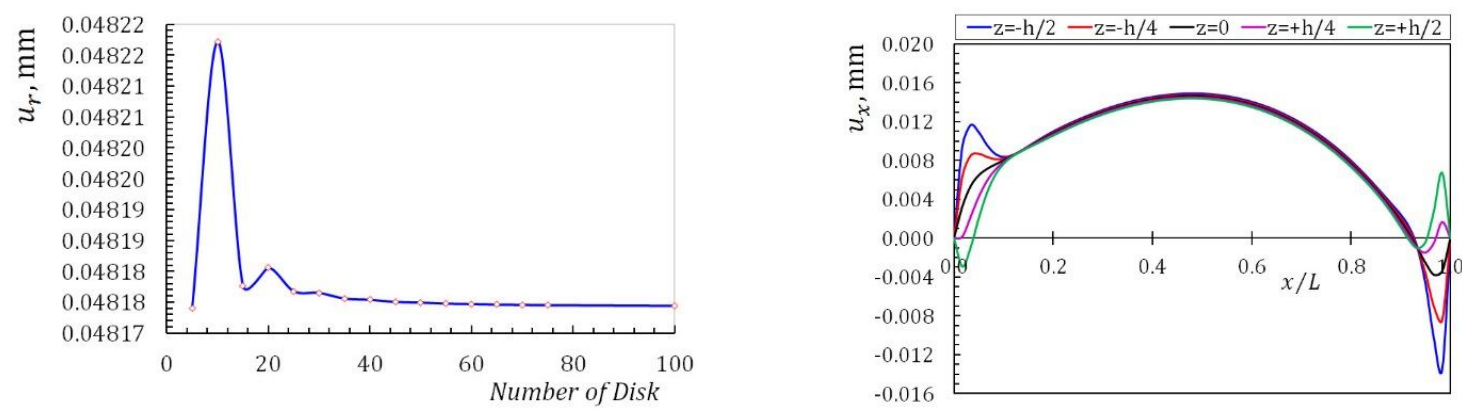

Fig. 4. Effect of the number of disk layers on the radial Fig. 5. Axial displacement distribution in different layers displacement

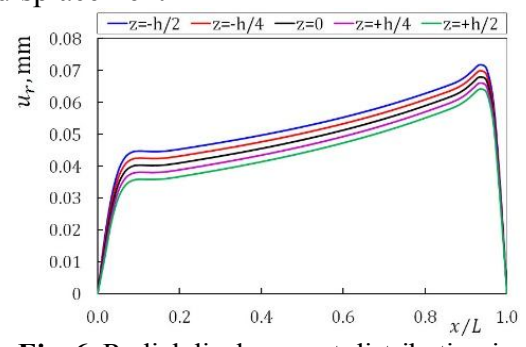

Fig. 6. Radial displacement distribution in different layers

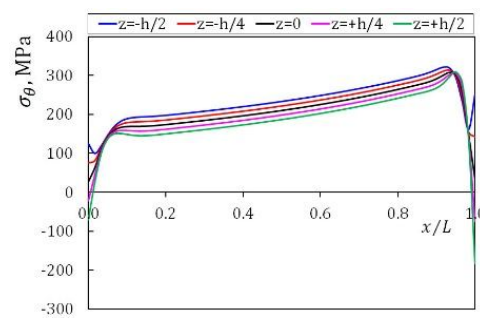

Fig. 7. Circumferential stress distribution in different layers

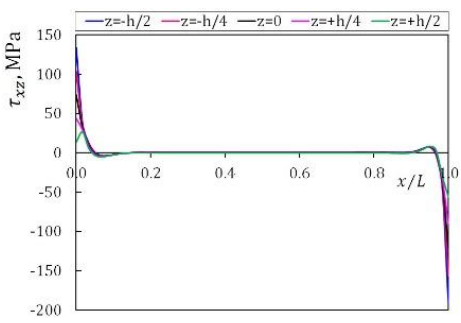

Fig. 8. Shear stress distribution in different layers 
Distribution of circumferential stress in various layers is shown in Fig. 7. The circumferential stress at all points depends on radius and length. The circumferential stress at layers close to the external surface is negative, and at other layers is positive. The greatest circumferential stress occurs in the internal surface $(z=-h / 2)$. Fig. 8 demonstrates the distribution of shear stress at different layers. The shear stress at points away from the boundaries at different layers is the same and trivial. However, at points near the boundaries, the stress is significant, especially in the internal surface, which is the greatest. In the Figs. 9-13, displacement and stress distributions are obtained using multilayer method (ML) and compared with the solutions of FEM. Figs. 9 to 13 show that the disk layer method based on FSDT has an acceptable amount of accuracy when one wants to obtain radial displacement, radial stress and circumferential stress. However, they are not useful for axial stress and not useful at all for radial displacement. It is possible to compensate for this by increasing the order of shear deformation theory.

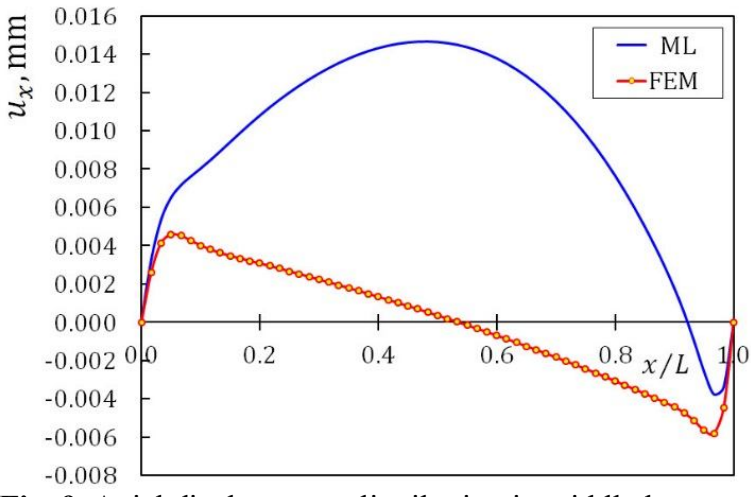

Fig. 9. Axial displacement distribution in middle layer

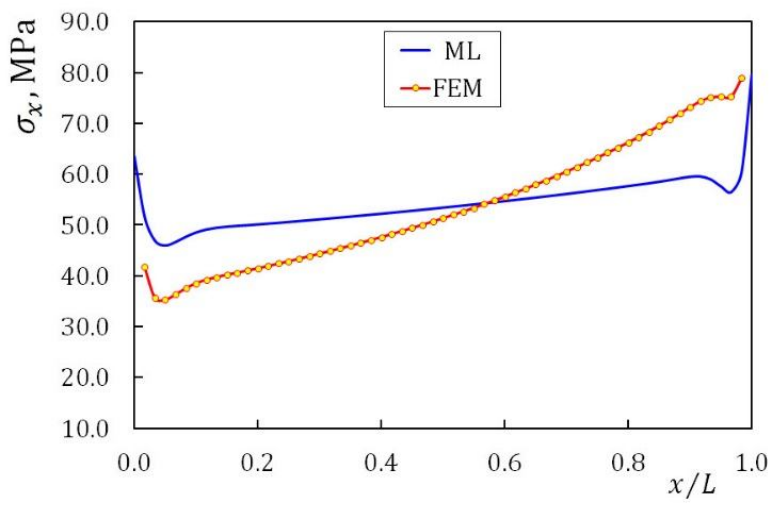

Fig. 11. Axial stress distribution in middle layer

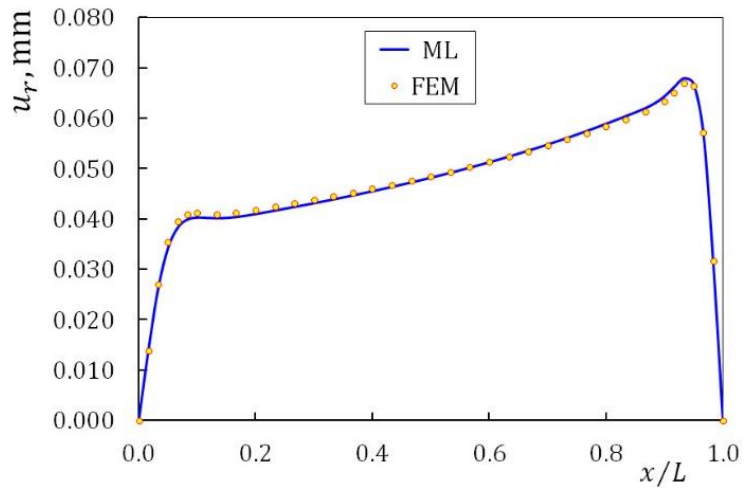

Fig. 10. Radial displacement distribution in middle layer

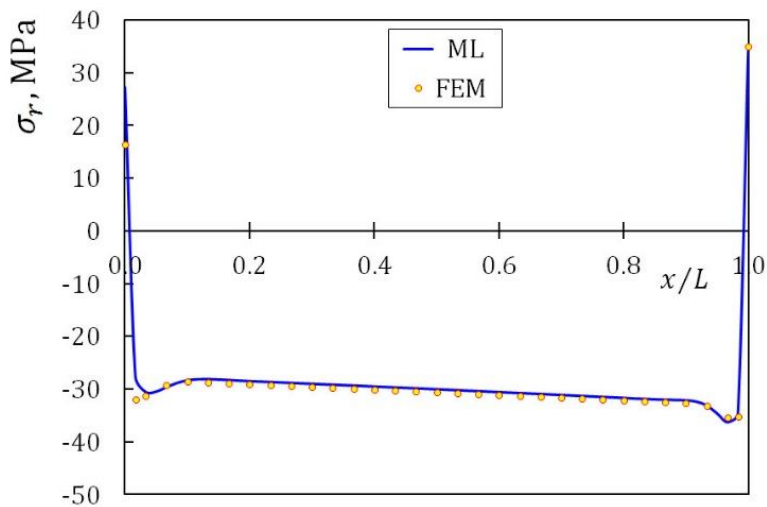

Fig. 12. Radial stress distribution in middle layer

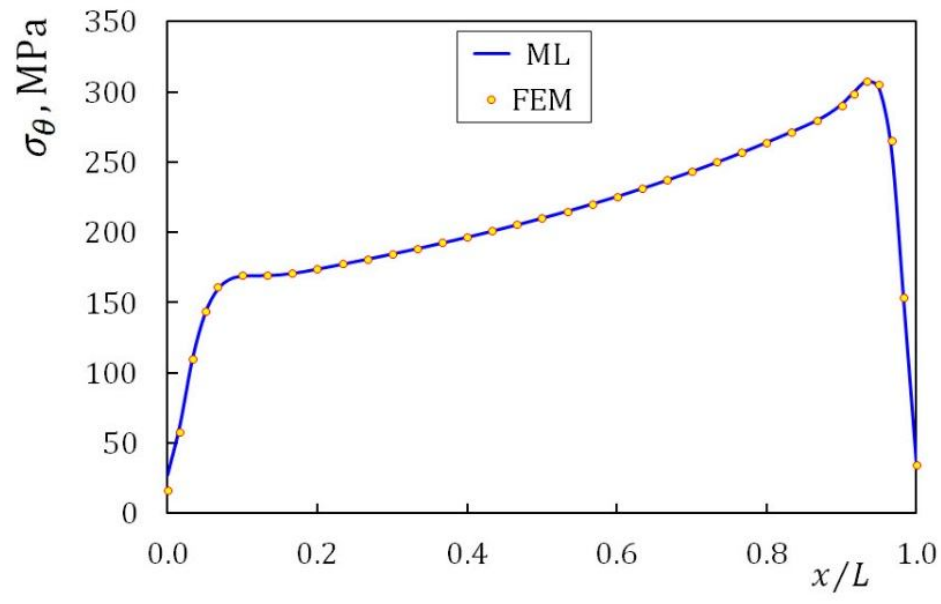

Fig. 13. Circumferential stress distribution in middle layer 
In Table 1, the values of stresses and displacements resulting from analysis of thick cylindrical shell with variable thickness through ML, MAM and FEM for clamped-clamped condition under uniform internal pressure in the middle layer have been presented.

Table 1. Comparison of values of ML, FEM and MAM

\begin{tabular}{cccccc}
\hline Method & $u_{r}, m m$ & $u_{x}, m m$ & $\sigma_{r}, M P a$ & $\sigma_{x}, M P a$ & $\sigma_{\theta}, M P a$ \\
\hline ML & 0.04817 & 0.0146 & -30.03 & 53.41 & 210.03 \\
FEM & 0.04846 & 0.0004 & -30.59 & 51.33 & 210.24 \\
MAM & 0.04832 & 0.0002 & -30.16 & 51.42 & 209.79 \\
\hline
\end{tabular}

\section{Conclusions}

In the present study,

(1): based on FSDT and elasticity theory, the governing equations of thick-walled disks were derived.

(2): Thick cylindrical shells with variable thickness were divided into disks with constant height.

(3): With considering continuity between layers and applying boundary conditions, the governing set of differential equations with constant coefficients was solved.

(4): The results obtained for stresses and displacements are compared with the analytical solutions and the solutions carried out through the FEM. Good agreement was found among the results.

Adventures of the semi-analytical using disk form multilayers are

$\checkmark$ First shear deformation theory and perturbation theory result in the analytical solution of the problem with higher accuracy and within a shorter period of time.

$\checkmark$ The solutions are not complicated and time-consuming.

$\checkmark$ The shells with different geometries and different loadings and different boundary conditions, with even variable pressure, could be more easily solved.

$\checkmark$ The method is very suitable for the purpose of calculation of radial stress, circumferential stress, shear stress and radial displacement.

Due to complex mathematical relations existing for analytical methods, governing them cannot be easily solved. Therefore, the multilayer disc form method can be considered as a good replacement for the analysis of thick-walled shells.

\section{References}

Eipakchi, H. R., Rahimi, G. H., \& Khadem, S. E. (2003). Closed form solution for displacements of thick cylinders with varying thickness subjected to non-uniform internal pressure. Structural Engineering and Mechanics, 16, 731-748.

Ghannad, M., \& Nejad, M. Z. (2010). Elastic analysis of pressurized thick hollow cylindrical shells with clamped-clamped ends. Mechanika, 5(85), 11-18.

Ghannad, M., Nejad, M. Z., \& Rahimi, G. H. (2009). Elastic solution of axisymmetric thick truncated conical shells based on first-order shear deformation theory. Mechanika, 5(79), 13-20.

Ghannad, M., Rahimi, G. H., \& Nejad, M. Z. (2012). Determination of displacements and stresses in pressurized thick cylindrical shells with variable thickness using perturbation technique. Mechanics, $18(1), 14-21$. 
Greenspon, J. E. (1960). Vibrations of a Thick-Walled Cylindrical Shell-Comparison of the Exact Theory with Approximate Theories. The Journal of the Acoustical Society of America, 32(5), 571578

Kang, J. H., \& Leissa, A. W. (2001). Three-dimensional field equations of motion, and energy functionals for thick shells of revolution with arbitrary curvature and variable thickness. Journal of applied mechanics, 68(6), 953-954.

Mirsky, I., \& Hermann, G. (1958). Axially motions of thick cylindrical shells, Journal of Applied Mechanics-Transactions of the ASME, 25, 97-102.

Naghdi, P. M., \& Cooper, R. M. (1956). Propagation of elastic waves in cylindrical shells, including the effects of transverse shear and rotary inertia, Journal of the Acoustical Society of America, 29, 56-63.

Nejad, M. Z., Rahimi, G. H., \& Ghannad, M. (2009). Set of field equations for thick shell of revolution made of functionally graded materials in curvilinear coordinate system. Mechanika, 3(77), 18-26.

Suzuki, K., Konno, M., \& Takahashi, S. (1981). Axisymmetric vibrations of a cylindrical shell with varying thickness. Bulletin of JSME, 24, 2122-2132.

Talaee, M., Alizadeh, M., \& Bakhshandeh, S. (2014). An exact analytical solution of non-Fourier thermal stress in cylindrical shell under periodic boundary condition. Engineering Solid Mechanics, 2(4), 293-302.

Vekua, I. N. (1965). The theory of thin shallow shells of variable thickness. In Proceedings of $A$. Razmadze Institute of Mathematics of Georgian Academy of Sciences, 30, 5-103.

Vlachoutsis, S. (1992). Shear correction factors for plates and shells. International Journal for Numerical Methods in Engineering, 33(7), 1537-1552. 